\title{
Genetic Heterogeneity in GJB2, COL4A3, ATP6V1B1 and EDNRB Variants Detected Among Hearing Impaired Families in Morocco
}

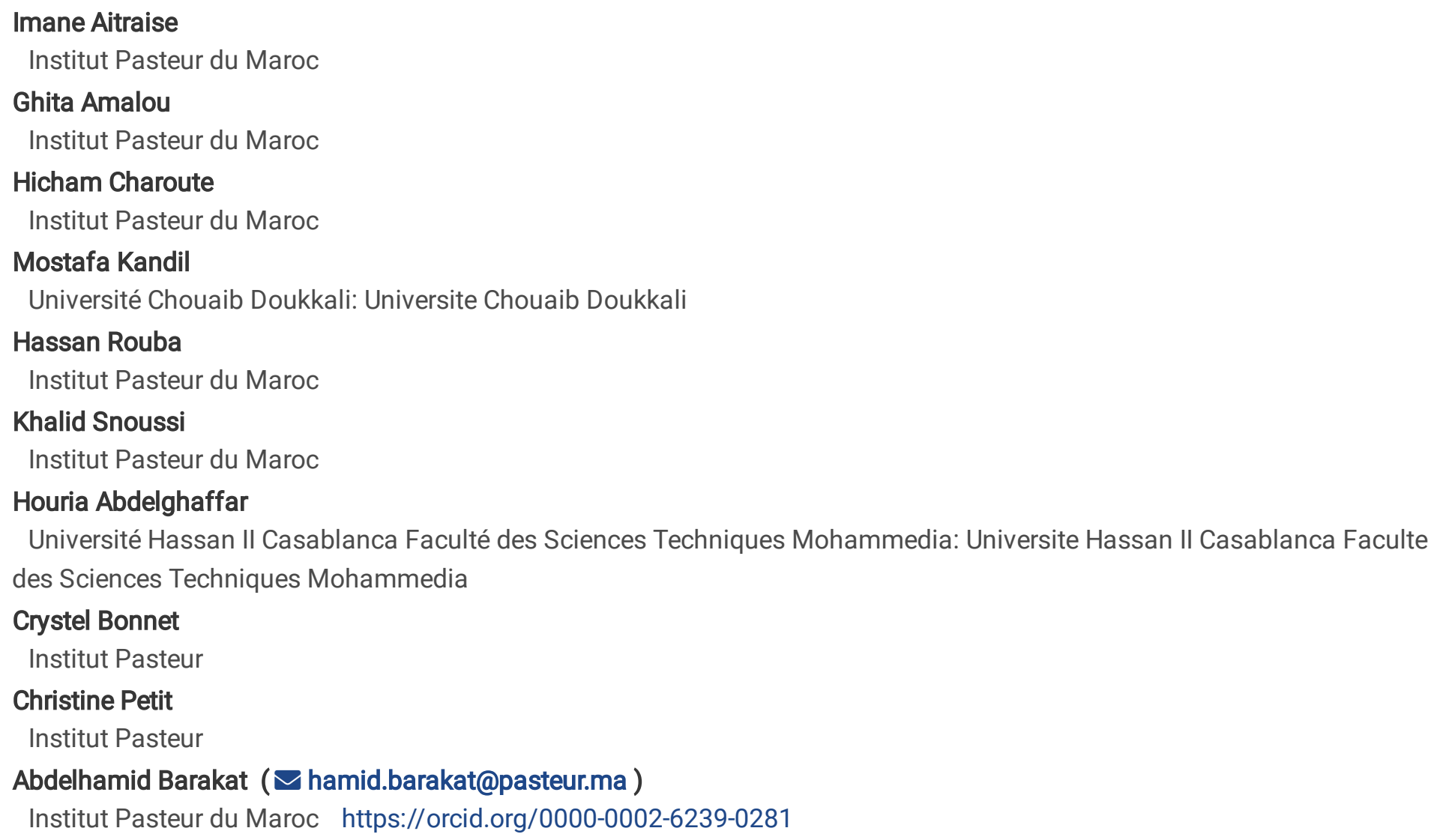

\section{Research Article}

Keywords: Whole Exome Sequencing, Mutation, Hearing Loss, Moroccan patients

Posted Date: August 30th, 2021

DOI: https://doi.org/10.21203/rs.3.rs-814770/v1

License: (c) (i) This work is licensed under a Creative Commons Attribution 4.0 International License. Read Full License

Version of Record: A version of this preprint was published at Molecular Biology Reports on March 17th, 2022. See the published version at https://doi.org/10.1007/s11033-022-07245-z. 


\section{Abstract}

Deafness has a very variable disease. It may occur as a result of external auditory canal involvement or a deficiency in the sound conduction mechanism (transmission deafness) or impairment of the cochlear, cochlear nerve or central auditory perception. Genetics is the most common cause, as approximately $70 \%$ of hearing disorders are of hereditary origin. $1 / 3$ of hereditary deafness is syndromic (associated with other symptoms) and $2 / 3$ are non-syndromic (isolated deafness). At this date, 173 loci of deafness gene have been reported in the literature (69 DFNA, 94 DFNB, 6 X-linked DFN, 2 DFNM, 1 DFNY and 1 AUNA1). For syndromic deafness, approximately 400 syndromes associated with hearing disorders are already described. Thus, the determination of causal mutations is a valuable aid for accurate and early diagnosis. This makes it possible to better guide the management since forms of deafness respond better to the cochlear implant than others. The correct diagnosis also gives an idea of the evolutionary profile of deafness and whether it is a syndromic deafness requiring special surveillance. In this study, we have examined the genetic causes of sensorineural hearing loss in Moroccan patients through whole exome sequencing (WES) to identify candidate genes for six severely deaf Moroccan families. The results revealed four genetic variants in the genes GJB2, COL4A3, ATP6V1B1 and EDNRB, which are therefore common causes of syndromic and non-syndromic deafness.

\section{Introduction}

The global population living with disabling hearing impairment is approximately $6.1 \%$ as estimated by the WHO recently [1]. Hearing loss is the most common human neurosensory defect in the world, occurring in approximately 1 in 1000 newborns, and $70 \%$ of these cases have a genetic etiology [2]. Hereditary hearing loss is transmitted in several ways: autosomal recessive, autosomal dominant, $\mathrm{X}$ - or Y-linked, and mitochondrial. They can be either isolated (non-syndromic) or associated with damage to other organs (syndromic) [3]. The GJB2 gene is the main cause of hereditary non-syndromic deafness in Morocco. Several mutations in this gene have been described as causing autosomal recessive and more rarely dominant hereditary deafness. The most prominent mutation in Morocco and the Mediterranean region is the deletion c.35delG [4]. The second cause of deafness in Morocco is the presence of a substitution c.242G > A located on LRTOMT gene [5]. Among the syndromic genes linked to deafness and which occur in the Moroccan population MYO7A [6], PEX1 [7], ADGRV1[8] and many others. For non-syndromic genes, there is TMC1 [9], CLDN14 [2] and PJVK [10]. These genes play a very important role in maintaining the normal physiology of the inner ear, each mutation of these can affect normal hearing physiology [11].

The genetic diagnosis of hearing loss is very important for the clinical evaluation of deaf persons and their families. Next Generation Sequencing (NGS) provides an opportunity to explore the genetic structure of the disease, and can be further be used as a benchmark for medical genetic testing [12].

In this study, Exome analysis was used to resolve the etiology of hearing loss in six Moroccan families.

\section{Patients And Methods}

In this study, we recruited six families with syndromic and no syndromic hereditary deafness SF193, SF55, SF175, SF181, SF177 et SF188. Family members were informed of the purpose of the study and gave their informed consent. The genetic study was approved by the medical ethics committee of the Morocco Pasteur Institute, and carried out in accordance with the protocol of the Helsinki declaration ( Fig. 1).

Whole exome sequencing (WES) was performed at integraGen (Evry.France). The Agilent Human exome V5 (50Mb) capture kit allows the capture of libraries, followed by paired end sequencing on Illumina Hiseq 2000. According to the manufacturer's protocol, the capture of the sequence was carried out. Using Illumina Real-Time Analysis Pipeline version 1.14 can generate an image analysis and call up databases using default parameters.

The short reads with paired ends were aligned against the human genome reference sequence hg19 (GRCH37). 
The bioinformatics analysis of the sequencing data was based on the Illumina pipeline (CASAVA.1.8). We have filtered the variants based on dbSNP (build132) and 1000 genome project databases. Finally, the functional effects of the novel variants were predicted using SIFT (Sorting Intolerant From Tolerant) and PolyPhen-2 (Polymorphism Phenotyping). For the six families analyzed, results were obtained for only four families.

To determine the segregation with the phenotype of the disease in these families, sanger sequencing was carried out to validate the mutation in the candidate gene. Specific primers have been designed using primer 3 (Table 1).

Table 1

Sequence of primers for allele-specific PCR for genes

\begin{tabular}{|lll|}
\hline Genes & Sequences & Product size \\
\hline GJB2 & F-AGAGTTGGTGTTTGCTCAGGA & $900 \mathrm{pb}$ \\
& R-GACTGAGCCTTGACAGCTGA & \\
& F-AGAACCTTCCAAGCTCCCTG & $383 \mathrm{pb}$ \\
EDNRB & R-GTCTCCCCAGCCATGTAGAA & \\
& R-CACTTCGGTTCCACTTCACA & $398 \mathrm{pb}$ \\
ATP6V1B1 & F-TGTTAGGAATGTGTGTGGGG & $567 \mathrm{pb}$ \\
& R-ACAATTTGGGGACAGGGG & \\
\hline
\end{tabular}

The 3D structures of the native and mutant EDNRB protein were predicted using SWISS Model, a fully automated protein structure homology-modelling server [13]. The predicted models were generated based on the structure of the Endothelin type B receptor in complex with Endothelin-3 (PDB ID: 6IGK). Targets (native and mutated EDNRB proteins) and template proteins share more than $94 \%$ of sequence similarity. The obtained structures were minimized using the Yasara Energy Minimization Server [14]. Amino acids interactions analysis and visualization were performed using YASARA software [15]. We used the following bioinformatics tools mCSM[16], SDM[17], DUET[18] et DeepDDG[19] to analyze the impact of amino acid substitutions on the stability of the EDNRB protein structure.

\section{Results}

\section{Syndromic genes}

For syndromic families, we found SF181.05 family suffering from deep congenital prelingual bilateral deafness with Waardenburg syndrome since the patient presents the following signs: pigment deficits, scratching, green eyes and some white hair. WES results revealed a novel homozygous mutation c.1225C > T;p.(Arg409Trp) of the EDNRB gene. Sanger sequencing confirmed that the affected patient SF181.05 was homozygous for the mutation, while the father and the other family members were heterozygous.

The c. $1225 \mathrm{C}>\mathrm{T}$ missense mutation has been predicted to be damaging, possibly damaging using the SIFT, FATHMM-MKL and POLYPHEN programs, deleterious by LRT software and disease causing by MutationTaster (Table 2). 
Table 2

Characteristics of the mutations

\begin{tabular}{|llllllll|}
\hline Genes & $\begin{array}{l}\text { DNA } \\
\text { Change }\end{array}$ & $\begin{array}{l}\text { Amino acid } \\
\text { variation }\end{array}$ & Polyphen & SIFT & MutationTaster & $\begin{array}{l}\text { FATHMM- } \\
\text { MKL }\end{array}$ & LRT \\
\hline EDNRB & $\begin{array}{l}\text { c.1225C }> \\
\text { T }\end{array}$ & p.Arg409Trp & $\begin{array}{l}\text { Probably } \\
\text { Damaging }\end{array}$ & Damaging & $\begin{array}{l}\text { Disease } \\
\text { causing }\end{array}$ & Damaging & Deleterious \\
\hline GJB2 & c.551G $>$ A & p.Arg184GIn & $\begin{array}{l}\text { Probably } \\
\text { Damaging }\end{array}$ & Damaging & $\begin{array}{l}\text { Disease } \\
\text { causing }\end{array}$ & Damaging & Deleterious \\
\hline COL4A3 & $\begin{array}{l}\text { c.3829G }> \\
\text { A }\end{array}$ & p.Gly1277Ser & $\begin{array}{l}\text { Probably } \\
\text { Damaging }\end{array}$ & Damaging & $\begin{array}{l}\text { Disease } \\
\text { causing }\end{array}$ & Damaging & Deleterious \\
\hline
\end{tabular}

Moreover, the alignment of multiple sequences of orthologous EDNRB proteins of different species showed that the missense mutation p.Arg409Trp affected a highly conserved residue (Fig. 2).

To estimate the potential structural impact of the p.Arg409Trp we carried out a molecular modelling study. The amino acid interactions analyses showed that this missense mutation did not affect hydrogen bounds. However, it may disturb the hydrophobic interactions between the residue in 409 position and its adjacent amino acids, we observed that the interaction with Leu405 residue was replaced by Glu410 (Fig. 3). A protein stability analysis was performed to estimate the effect of p.Arg409Trp mutation of the protein stability. A destabilizing effect was predicted based on four different computational tools (Table 3).

Table 3

Mutation effects on EDNRB 3D structure stability

\begin{tabular}{|lll|}
\hline Software & Prediction & Stability change $(\Delta \Delta \mathrm{G}: \mathrm{Kcal} / \mathrm{mol})$ \\
\hline mCSM & Destabilizing & -0.017 \\
SDM & Destabilizing & -0.31 \\
\hline DUET & Destabilizing & -0.31 \\
\hline DeepDDG & Destabilizing & -0.369 \\
\hline
\end{tabular}

The SF193 is a consanguineous, congenital family, with profound bilateral deafness, medullary nephrocalcinosis, stratroponderal delay and distal tubular acidosis. In the index case (SF193.03) of the SF193 family, an insertion of the ATP6V1B1 gene NM-001692.4: c.1155dupC;p.(Ile386HisfsTer56) has been identified by WES. Sanger sequencing confirmed that the affected patient (SF193.03) was homozygous for the mutation, while the unaffected father (SF193.01) and healthy mother (SF193.02) were heterozygous for this mutation.

The SF175 family is characterized by isolated, bilateral, prelingual deafness and onset of myopia. After the analysis done, we found the COL4A3 gene which is already described in deafness and also in Alport syndrome. WES results showed homozygous missense mutation (c.3829G > A; p.(Gly1277Ser)) in COL4A3 gene for the deaf index patient (SF175.03) and heterozygous for the normal sister (SF175.04) and the parents confirmed by sanger sequencing (Table 4).

\section{Non-syndromic genes}

The SF55 family has severe deafness. The results showed that heterozygous mutations were found in the already described GJB2 gene and were involved in a dominant form. Sanger sequencing confirmed that the affected patient (SF55.04) is heterozygous for the missense mutation (c.551G > A;p. (Arg184GIn)), while the mother (SF55.02) is homozygous wild-type, and the father (SF55.01) and brother (SF55.04) are heterozygous (Table 4). 
Table 4: Identity of the four pathogenic homozygous variants found

by WES of DNA

\begin{tabular}{|lllllll|}
\hline Genes & rs & Location & Reference sequence & DNA Change & Amino acid variation & MAF Gnomad \\
\hline GJB2 & rs80338950 & Exon 2 & NM_004004.6 & c.551G>A & p.Arg184Gln & - \\
\hline COL4A3 & rs190598500 & Exon 43 & NM_000091.5 & c.3829G>A & p.Gly1277Ser & $3.63 \mathrm{e}-4$ \\
\hline ATP6V1B1 & rs781969081 & Exon 12 & NM_001692.4 & c.1155dup & p.Ile386Hisfs*56 & $3.22-5$ \\
\hline EDNRB & rs200363611 & Exon 6 & NM_001201397.1 & c.1225C>T & p.Arg409Trp & $1.78 \mathrm{e}-4$ \\
\hline
\end{tabular}

\section{Discussion}

In this work, we describe two genes that has been described in the Moroccan population, complete sequencing of the exome revealed a new mutation described in Morocco for the first time in the GJB2 gene, which is already identified as being the main cause of hereditary non-syndromic deafness in Morocco affecting around 35\% of cases. It codes for connexin 26, a protein found in communicating junctions or gap junction [4]. The most prominent mutation in Morocco and around the Mediterranean is the deletion of $\mathrm{G}$ at position 35 on the DNA encoding written c.35delG, usually in our laboratory the c.35delG mutation is always initially sought by direct sequencing as the first step in molecular diagnosis. Other mutations in this gene have been described as causing autosomal recessive hereditary deafness while dominant inheritance rarely occurs. The $p$. R184Q mutation cited in our study which was observed for the first time in the Moroccan population. According to [20] this mutation was also observed for the first time in the Indian population without any associated syndrome, and it was also found in the province of Jiangsu [21].

The second gene ATP6V1B1 encodes the vacuolar $\mathrm{H}+$ ATPase $\mathrm{B} 1$ subunit located on the apical surface of alpha intercalated cells in the distal tubule, and is also expressed in epithelium of the human cochlea and the endolymphatic sac [22]. People with ATP6V1B1 mutations have the most common sensorineural hearing loss, the homozygous variant c. 1155dupC in ATP6V1B1 causes a change in the reading frame of isoleucine 386 introducing a premature stop codon. This variant of ATP6V1B1 was found in a homozygous patient from a Mexican family [23], two Moroccan patients [22], and Tunisian children with recessive form of dRTA associated to precocious hearing loss [24].

Besides to the usual genes previously published in Morocco, we have described here the first mutation of the COL $4 A 3$ gene in the Moroccan population affected by deafness. Mutations in the COL4A3 gene are linked to kidney problems, deafness, and eye damage known as Alport syndrome [25]. Fallerini et al have already described the p.Gly1277Ser variant in COL4A3 in 3 families in Italy with Alport syndrome, and was found here in a Moroccan family for the first time with hearing loss and onset of myopia and no kidney problem for the moment but probably because the patient is still very young. They also found this mutation in the heterozygous state in a patient with the autosomal dominant form [26].

We also found a novel mutation in the gene $\operatorname{EDNRB}(\mathrm{c} .1225 \mathrm{C}>\mathrm{T})$ which causes a substitution of arginine at position 409 by tryptophan and is reported in the EXAC database (rs 200363611) with a frequency of 0.000025 / 3. EDNRB gene mutations are linked to Waardenburg de type IV (WS4) [27]. Other studies [28, 29] have suggested that EDNRB should be considered as another pathogenic gene prevalent in WS type 1 to the heterozygous state, whereas[27] estimated that the EDNRB mutations are responsible for $5-6 \%$ of WS type 2 .

A molecular modeling study was performed to evaluate the structural impact of the p.Arg409Trp missense mutation. This mutation appears to cause a change in hydrophobic interactions, which resulted in a change in the 3D structure of the EDNRB protein between the native state and the mutated state. In addition, this mutation has a destabilizing effect on the stability of proteins. 
In conclusion, this study describes the involvement of genetic variants of the GJB2, COL4A3, EDNRB and ATP6V1B1 genes in syndromic and non-syndromic deafness in Moroccan patients. These results can be considered very important because they allow to show even more the genetic diversity of hereditary deafness in Moroccan patients. In addition, they also make it possible to enrich national and international data concerning the Moroccan population.

\section{Declarations}

\section{ACKNOWLEDGEMENTS}

The authors are indebted to the families who contributed to this study. This project was supported by the Institut Pasteur du Maroc (IPM).

\section{FUNDING}

No funds, grants, or other support was received.

\section{CONFLICT OF INTEREST}

The authors declare that they have no conflict of interest.

\section{AVAILABILITY OF DATA AND MATERIAL}

Data will be provided by the authors upon request.

\section{CODE AVAILABILITY}

Not applicable

\section{AUTHOR CONTRIBUTIONS}

Conceptualization: [Houria Abdelghaffar], [Abdelhamid Barakat] and [Mostafa Kandil] ; Formal analysis: [Imane Ait Raise], [Ghita Amalou] ; Funding acquisition: [Abdelhamid Barakat], [Hassan Rouba]; Investigation: [Imane Ait Raise], [Ghita Amalou]; Methodology: [Imane Ait Raise], [Ghita Amalou], [Crystel Bonnet] ; Resources: [Khalid Snoussi], [Christine Petit] and [Abdelhamid Barakat]; Software: [Imane Ait Raise], [Hicham Charoute]; Writing - original draft: [Imane Ait Raise], [Ghita Amalou]; Writing - review \& editing: [Houria Abdelghaffar], [Crystel Bonnet], and [Abdelhamid Barakat].

\section{ETHICS APPROVAL}

The genetic study was approved by the medical ethics committee of the Morocco Pasteur Institute.

\section{CONSENT TO PARTICIPATE}

Informed consent was obtained from legal guardians.

\section{CONSENT TO PUBLISH}

Patients signed informed consent regarding publishing their data.

\section{References}

1. Olusanya BO, Davis AC, Hoffman HJ (2019) Hearing loss: rising prevalence and impact. Bull World Health Organ 97:646646A. https://doi.org/10.2471/BLT.19.224683 
2. Charif M, Bakhchane A, Abidi O, et al (2013) Analysis of CLDN14 gene in deaf Moroccan patients with non-syndromic hearing loss. Gene 523:103-105. https://doi.org/10.1016/j.gene.2013.03.123

3. Marlin S, Jonard L, Feldmann D, et al (2009) [Genetic deafness in adults]. Rev Prat 59:630-631

4. Bakhchane A, Bousfiha A, Charoute H, et al (2016) Update of the spectrum of GJB2 gene mutations in 152 Moroccan families with autosomal recessive nonsyndromic hearing loss. Eur J Med Genet 59:325-329.

https://doi.org/10.1016/j.ejmg.2016.05.002

5. Charif M, Bounaceur S, Abidi O, et al (2012) The c.242G>A mutation in LRTOMT gene is responsible for a high prevalence of deafness in the Moroccan population. Mol Biol Rep 39:11011-11016. https://doi.org/10.1007/s11033-012-2003-3

6. Bakhchane A, Charif M, Bousfiha A, et al (2017) Novel compound heterozygous MYO7A mutations in Moroccan families with autosomal recessive non-syndromic hearing loss. PloS One 12:e0176516. https://doi.org/10.1371/journal.pone.0176516

7. Bousfiha A, Bakhchane A, Charoute $\mathrm{H}$, et al (2017) A novel PEX1 mutation in a Moroccan family with Zellweger spectrum disorders. Hum Genome Var 4:17009. https://doi.org/10.1038/hgv.2017.9

8. Bousfiha A, Bakhchane A, Charoute H, et al (2017) Novel compound heterozygous mutations in the GPR98 (USH2C) gene identified by whole exome sequencing in a Moroccan deaf family. Mol Biol Rep 44:429-434. https://doi.org/10.1007/s11033017-4129-9

9. Bakhchane A, Charoute $\mathrm{H}$, Nahili $\mathrm{H}$, et al (2015) A novel mutation in the TMC1 gene causes non-syndromic hearing loss in a Moroccan family. Gene 574:28-33. https://doi.org/10.1016/j.gene.2015.07.075

10. Salime S, Charif M, Bousfiha A, et al (2017) Homozygous mutations in PJVK and MY015A genes associated with nonsyndromic hearing loss in Moroccan families. Int J Pediatr Otorhinolaryngol 101:25-29.

https://doi.org/10.1016/j.ijporl.2017.07.024

11. Shearer AE, Hildebrand MS, Smith RJ (1993) Hereditary Hearing Loss and Deafness Overview. In: Adam MP, Ardinger HH, Pagon RA, et al (eds) GeneReviews ${ }^{\circledR}$. University of Washington, Seattle, Seattle (WA)

12. Shearer AE, DeLuca AP, Hildebrand MS, et al (2010) Comprehensive genetic testing for hereditary hearing loss using massively parallel sequencing. Proc Natl Acad Sci U S A 107:21104-21109. https://doi.org/10.1073/pnas.1012989107

13. Biasini M, Bienert S, Waterhouse A, et al (2014) SWISS-MODEL: modelling protein tertiary and quaternary structure using evolutionary information. Nucleic Acids Res 42:W252-258. https://doi.org/10.1093/nar/gku340

14. Krieger E, Joo K, Lee J, et al (2009) Improving physical realism, stereochemistry, and side-chain accuracy in homology modeling: Four approaches that performed well in CASP8. Proteins 77 Suppl 9:114-122. https://doi.org/10.1002/prot.22570

15. Krieger E, Vriend G (2014) YASARA View - molecular graphics for all devices - from smartphones to workstations. Bioinforma Oxf Engl 30:2981-2982. https://doi.org/10.1093/bioinformatics/btu426

16. Pires DEV, Ascher DB, Blundell TL (2014) mCSM: predicting the effects of mutations in proteins using graph-based signatures. Bioinforma Oxf Engl 30:335-342. https://doi.org/10.1093/bioinformatics/btt691

17. Pandurangan AP, Ochoa-Montaño B, Ascher DB, Blundell TL (2017) SDM: a server for predicting effects of mutations on protein stability. Nucleic Acids Res 45:W229-W235. https://doi.org/10.1093/nar/gkx439

18. Pires DEV, Ascher DB, Blundell TL (2014) DUET: a server for predicting effects of mutations on protein stability using an integrated computational approach. Nucleic Acids Res 42:W314-319. https://doi.org/10.1093/nar/gku411 
19. Cao H, Wang J, He L, et al (2019) DeepDDG: Predicting the Stability Change of Protein Point Mutations Using Neural Networks. J Chem Inf Model 59:1508-1514. https://doi.org/10.1021/acs.jcim.8b00697

20. Pavithra A, Chandru J, Jeffrey JM, et al (2017) Rare compound heterozygosity involving dominant and recessive mutations of GJB2 gene in an assortative mating hearing impaired Indian family. Eur Arch Oto-Rhino-Laryngol Off J Eur Fed Oto-Rhino-Laryngol Soc EUFOS Affil Ger Soc Oto-Rhino-Laryngol - Head Neck Surg 274:119-125.

https://doi.org/10.1007/s00405-016-4229-5

21. Shi L, Chen J, Li J, et al (2016) Prevalence of GJB2 gene mutation in 330 cochlear implant patients in the Jiangsu province. J Laryngol Otol 130:902-906. https://doi.org/10.1017/S0022215116008689

22. Boualla L, Jdioui W, Soulami K, et al (2016) Clinical and molecular findings in three Moroccan families with distal renal tubular acidosis and deafness: Report of a novel mutation of ATP6V1B1 gene. Curr Res Transl Med 64:5-8. https://doi.org/10.1016/j.retram.2016.01.005

23. Escobar LI, Simian C, Treard C, et al (2016) Mutations in ATP6V1B1 and ATP6V0A4 genes cause recessive distal renal tubular acidosis in Mexican families. Mol Genet Genomic Med 4:303-311. https://doi.org/10.1002/mgg3.205

24. Elhayek D, Perez de Nanclares G, Chouchane S, et al (2013) Molecular diagnosis of distal renal tubular acidosis in Tunisian patients: proposed algorithm for Northern Africa populations for the ATP6V1B1, ATP6V0A4 and SCL4A1 genes. BMC Med Genet 14:119. https://doi.org/10.1186/1471-2350-14-119

25. Fallerini C, Baldassarri M, Trevisson E, et al (2017) Alport syndrome: impact of digenic inheritance in patients management. Clin Genet 92:34-44. https://doi.org/10.1111/cge.12919

26. Heidet L, Arrondel C, Forestier L, et al (2001) Structure of the human type IV collagen gene COL4A3 and mutations in autosomal Alport syndrome. J Am Soc Nephrol JASN 12:97-106

27. Issa S, Bondurand N, Faubert E, et al (2017) EDNRB mutations cause Waardenburg syndrome type II in the heterozygous state. Hum Mutat 38:581-593. https://doi.org/10.1002/humu.23206

28. Li W, Mei L, Chen H, et al (2019) New Genotypes and Phenotypes in Patients with 3 Subtypes of Waardenburg Syndrome Identified by Diagnostic Next-Generation Sequencing. Neural Plast 2019:7143458. https://doi.org/10.1155/2019/7143458

29. Morimoto N, Mutai H, Namba K, et al (2018) Homozygous EDNRB mutation in a patient with Waardenburg syndrome type 1. Auris Nasus Larynx 45:222-226. https://doi.org/10.1016/j.anl.2017.03.022

\section{Figures}



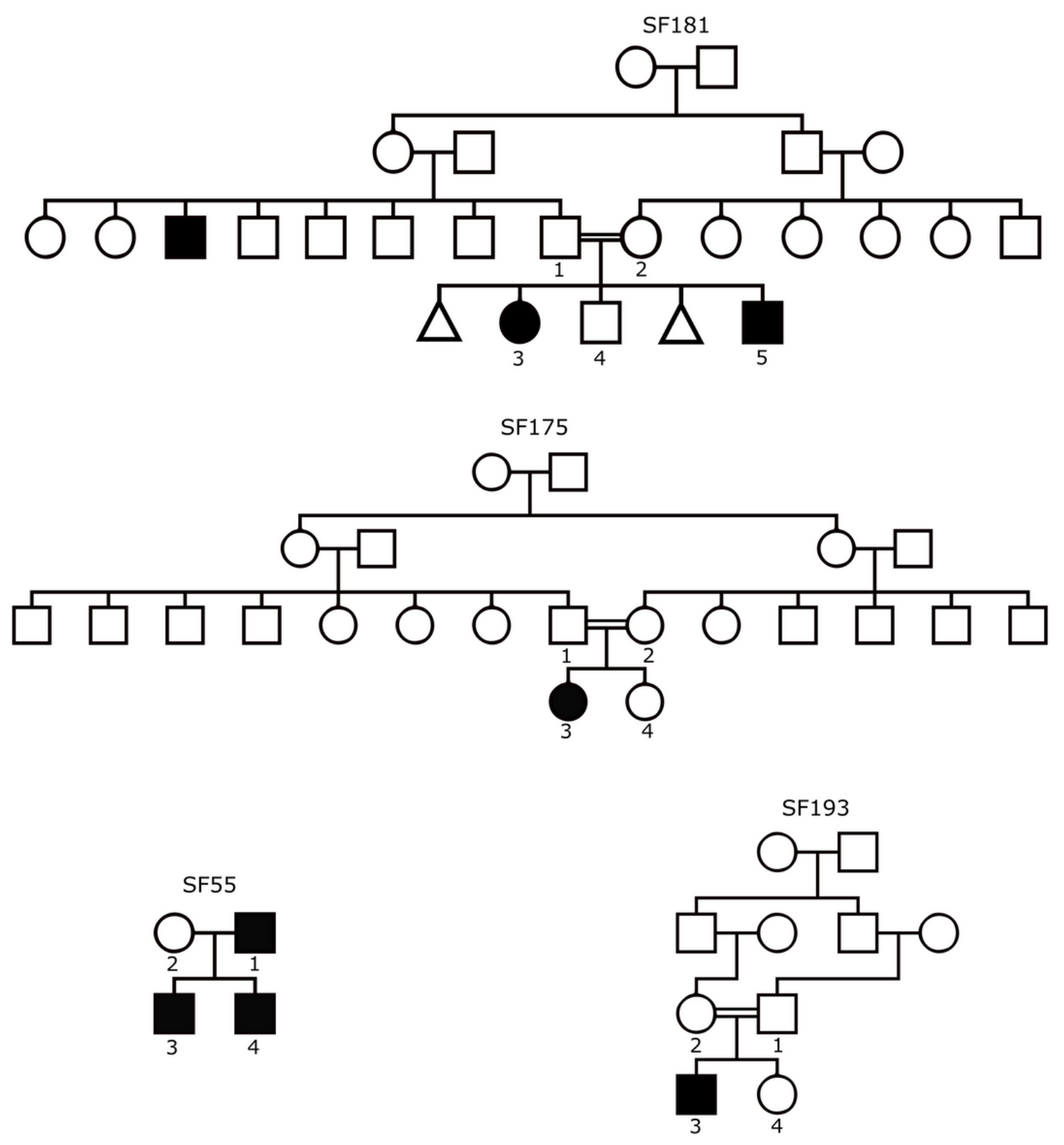

Figure 1

The pedigree of all the families 
Homo sapiens

Bos taurus

Rattus norvegicus

Mus musculus

Sus scrofa

Canis lupus familiaris

Oryctolagus cuniculus

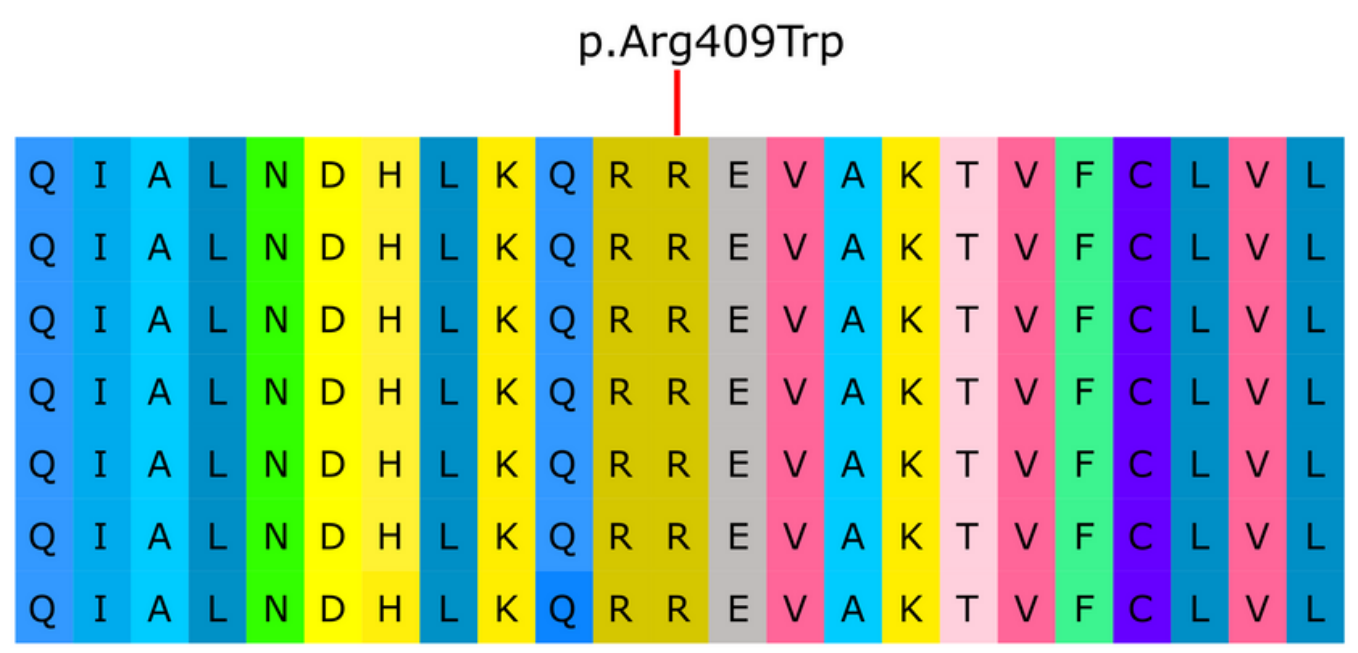

\section{Figure 2}

Alignment of EDNRB amino acid from different species 
b

a
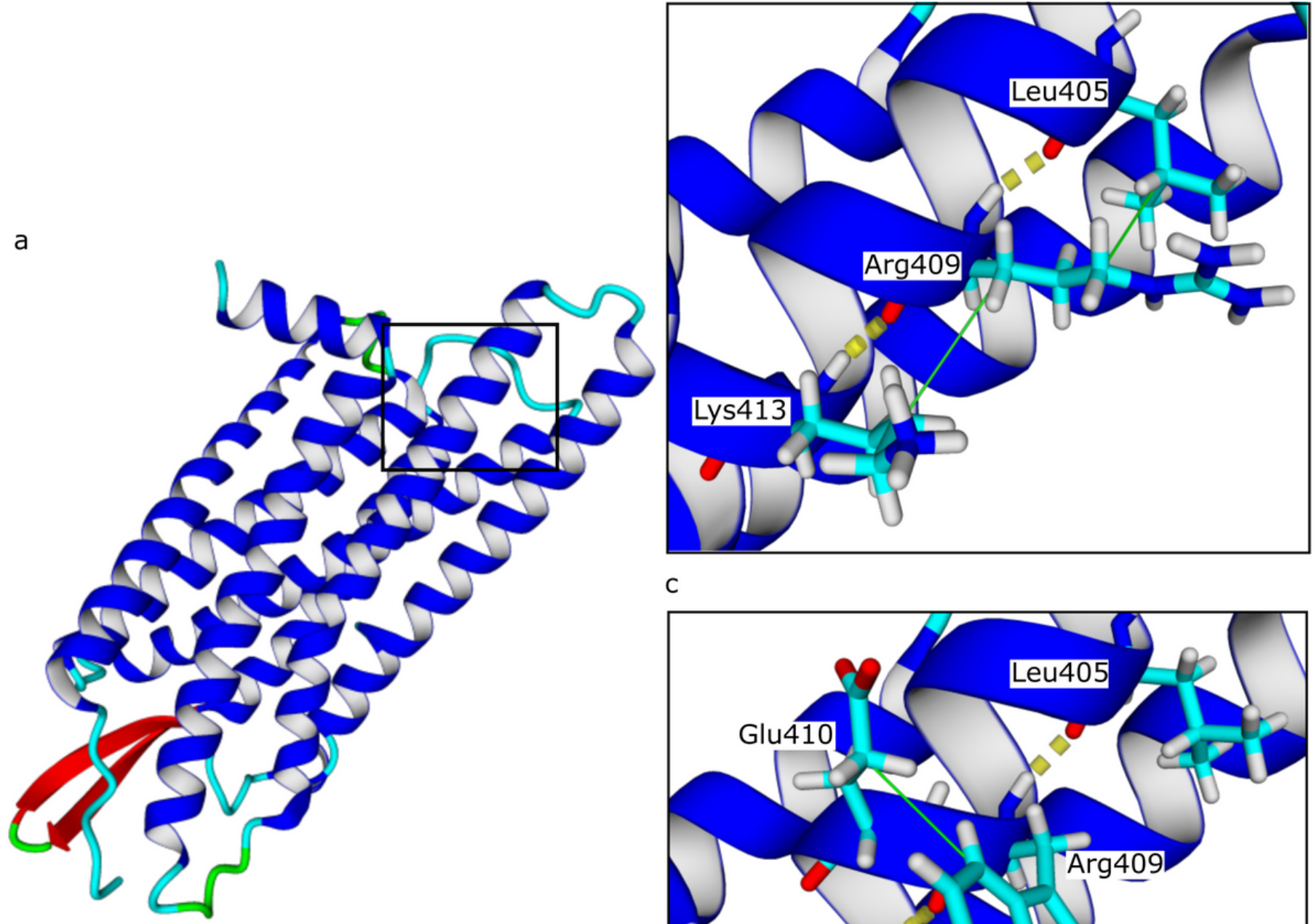

C

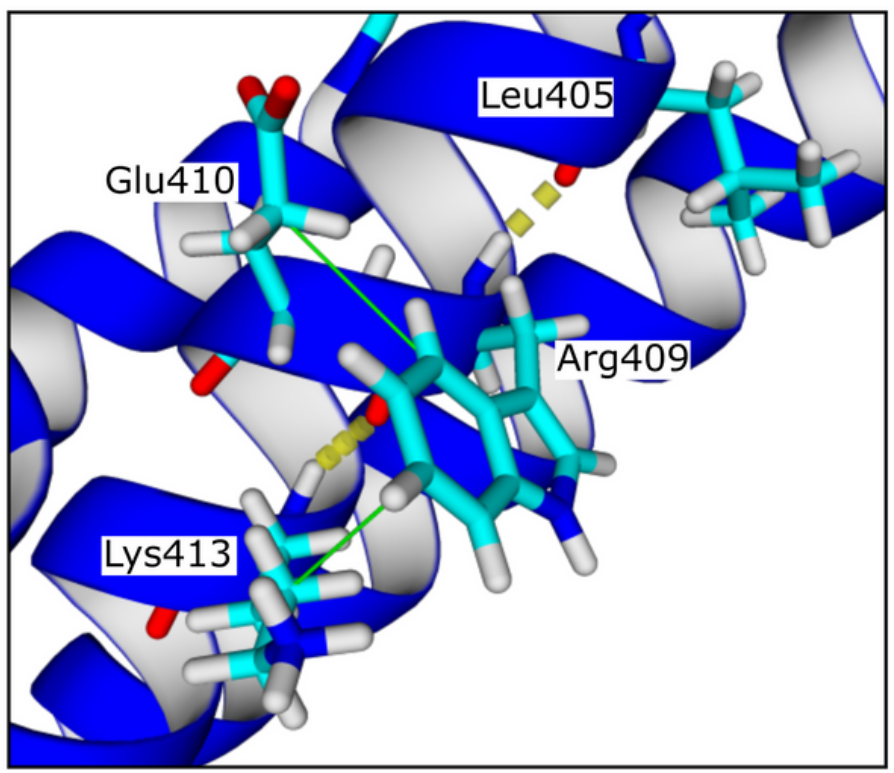

Figure 3

The potential structural impact of the p.Arg409Trp mutation of the EDNRB gene is revealed by molecular modeling. (a)Threedimensional structural modeling of EDNRB. (b, c) Hydrogen bonds and hydrophobic interactions predicted by Yasara software. Yellow dotted lines represent hydrogen bonds and green lines represent hydrophobic interactions. 